\title{
Envejecimiento saludable: perspectiva de género y de ciclo vital
}

\author{
Ma Pilar Montero, Ma Rosario López-Giménez, Paula \\ Acevedo y Ana I. Mora \\ Universidad Autónoma de Madrid (España)
}

\begin{abstract}
Objetivo: Identificar factores bioculturales y sociales que actúan en diferentes etapas del ciclo vital, implicados en la forma de envejecer de mujeres y hombres mayores de 65 años. Material y Método: 213 personas; edad media $73.9(s t d=5.8)$ en mujeres y 74.8 $(s t d=6.6)$ en hombres. Datos recogidos en Centros Culturales y de Ocio de la Comunidad de Madrid. El envejecimiento saludable (a partir del número de enfermedades diagnosticadas, percepción de la salud, satisfacción con la vida y estado auditivo y bucal), fue considerado como variable dependiente. La edad, sexo, nivel de estudios, talla sentado, envergadura, edad de primera maternidad y número total de hijos fueron consideradas como variables independientes. Se utilizaron modelos de regresión lineal múltiple para analizar la relación de estas variables con la calidad del envejecimiento. Resultados: El envejecimiento saludable se asocia negativamente con edad y directamente con talla sentado en hombres. En mujeres el envejecimiento saludable se asocia positivamente con años de educación, cuando se incluye la edad de primera maternidad, ésta es la única variable con efecto significativo. Conclusiones: Existen diferentes factores que actúan a lo largo de la vida de los hombres y de las mujeres y que afectan de manera distinta a su forma de envejecer.
\end{abstract}

Palabras clave: Envejecimiento saludable, género, patrones reproductores, estudios, envergadura, talla sentada.

Healthy aging: gender and lifecourse perspective cycle. Objective: To identify biocultural and social factors acting at different stages of lifecourse, involved in the form of aging in women and men over 65 years. Material and Methods: The sample consists of 213 individuals; mean age was 73.9 years $(s t d=5.8)$ for women and $74.8(s t d=6.6)$ for men. Data were collected in Cultural and Leisure Centers in the Community of Madrid. Healthy aging (created from the number of diagnosed diseases, perceived health, life satisfaction, hearing and oral status) was considered as the dependent variable. The independent variables considered in this study were age, sex, educational level, sitting height, spam, age at first maternity and the total number of children. Linear multiple regression models were used for statistical analysis. Results: Healthy aging was negatively associated with age and positively with the sitting height in men. In women, healthy aging was positively associated with years of education, when age at first motherhood is included in the model, this in the only variable remaining with a significant effect. Conclusions: There are several factors acting across the life of men and women and that affect differently the way women and men get old.

Keywords: Healthy Aging, gender, reproductive patterns, education level, arm-spam, sitting height.

Correspondencia: M. Pilar Montero-López. Departamento de Biología. Universidad Autónoma de Madrid. C/ Darwin, 2. C.P.: 28046. Madrid (España). E-mail: pilar.montero@uam.es 
El envejecimiento es un proceso biológico complejo y multifactorial, que se da en la última etapa del ciclo vital. Las mejoras de los sistemas sociales y sanitarios han ido logrando un retardo en el deterioro cronológico de los sistemas fisiológicos y cognitivos, y con ello un retraso de la edad de muerte (Adams y Whithe, 2004).

El aumento de la esperanza de vida y la tendencia al envejecimiento de la población mundial (Vaupel, 2010) tienen importantes consecuencias sociales, económicas y sanitarias (Christensen et al., 2009). En la actualidad, el reto no es solo conseguir más años de vida sino más calidad de vida que proporcione una adecuada calidad de vida a las personas y disminuya los costes que debe asumir la sociedad.

En 2002, la OMS propuso por primera vez el concepto de envejecimiento activo como un proceso óptimo de adaptación, que permite a las personas envejecer en buenas condiciones de salud, de funcionamiento físico y psicológico, de sentimientos positivos y de alta participación social (WHO, 2002).

Los indicadores de envejecimiento saludable y/o activo varían según el país o población que se analice (Montero y Rodríguez, 2013; Hank, 2010). En el caso de España, que tiene una elevada esperanza de vida (Salomon et al., 2013), se obtienen bajas prevalencias de envejecimiento activo, en comparación con otras poblaciones europeas. Montero y Rodríguez (2013) explican esta paradoja como resultado de las distintas circunstancias históricas vividas por los grupos de mayor edad.

Las diferencias sociales y económicas a las que se enfrentan las personas a lo largo del proceso de crecimiento y desarrollo, se pueden estimar indirectamente en etapas más tardías del ciclo vital mediante características morfológicas, como las relativas al tamaño final alcanzado por el individuo al finalizar su crecimiento (talla, envergadura y talla del tronco o "talla sentado"). Estas características están asociadas a su vez con la salud en la etapa adulta y condicionan el proceso de envejecimiento (Gularnik et al., 2006; Hayward y Gorman, 2004).

Algunos estudios sobre la calidad del envejecimiento mencionan la salud percibida y la satisfacción con la vida como buenos indicadores de envejecimiento (Montero y Rodríguez, 2013).

Dentro de la historia de vida, el género es una categoría de gran importancia en el estudio del proceso de envejecimiento y en la calidad del mismo. En el caso de las mujeres, las características del ciclo reproductivo y el efecto de la maternidad pueden ser también factores de influencia sobre los indicadores de envejecimiento saludable, tanto objetivos como subjetivos.

El objetivo de este estudio es identificar factores bioculturales y sociales que actúan en diferentes etapas del ciclo vital y que pueden afectar a la forma de envejecer de mujeres y hombres mayores de 65 años. Entre estos factores consideraremos indicadores antropométricos como la estatura (indicador de condiciones nutricionales y de exposición a enfermedades durante el crecimiento) y el nivel de estudios (indicador 
de condiciones socioeconómicas del individuo en su juventud). En el caso de las mujeres, además se analizará la relación entre patrones de envejecimiento y patrones reproductores (número de hijos y edad de maternidad).

\section{MATERIAL Y MÉTODO}

Se trata de un estudio con un doble diseño, transversal y retrospectivo. La muestra está formada por 213 personas (79 hombres, 37.1\%, y 134 mujeres, 62.9\%) de 65 a 89 años (media=73.9 años; $s t d=5.8)$ en mujeres y 74.8 años ( $s t d=6.6)$ en los hombres) residentes en la Comunidad Autónoma de Madrid. Los individuos fueron medidos y entrevistados por antropometristas e investigadoras, del Dpto. de Biología de la Universidad Autónoma de Madrid, en centros culturales y de ocio para personas mayores. Previamente, los participantes firmaron un consentimiento informado donde se garantizó la confidencialidad de los datos de acuerdo con las directrices establecidas en la Declaración de Helsinki. Se realizaron preguntas sobre variables socio-económicas, de salud percibida y patologías diagnosticadas.

Como indicador socioeconómico se utilizó el número de años de escolarización de la persona y el máximo nivel de estudios alcanzados. La percepción del estado de salud, se obtuvo mediante la pregunta "Comparado con otras personas de su misma edad, ¿considera que su salud es: 1. mala, 2. regular, 3. buena o 4. тиу buena?", también se preguntó sobre la satisfacción con la vida mediante la pregunta “ ¿en qué medida se siente satisfecho con su vida 1. muy poco, 2. algo, 3. bastante o 4. mucho?”.

La salud objetiva se valoró con el número de enfermedades diagnosticadas y el estado de su salud auditiva, visual y bucal. Así mismo, también se recogió información sobre la presencia de determinadas enfermedades como: hipertensión, hipercolesterolemia, enfermedades osteoarticulares, respiratorias, cardiovasculares, endocrinas, digestivas, genitourinarias, oncológicas, psicológicas, dérmicas y alergias.

Se creó la variable "envejecimiento saludable", mediante la suma de las puntuaciones de las categorías de las variables satisfacción con la salud, satisfacción con la vida, estado auditivo y estado bucal. Esta variable puede tomar valores entre 4 (correspondiente a peor envejecimiento) y 16 (correspondiente a mejor envejecimiento).

Se tomaron las siguientes medidas antropométricas directas talla $(\mathrm{cm})$, peso $(\mathrm{kg})$, talla sentada $(\mathrm{cm})$ y envergadura $(\mathrm{cm})$. A partir de la talla y el peso se calculó el Índice de Masa Corporal o Índice de Quetelet mediante la fórmula: IMC $=($ peso $) \mathrm{kg} / \mathrm{talla}$ $(\mathrm{m})^{2}$. Con todas estas variables se creó una base de datos que fue procesada estadísticamente con el programa SPSS 20.0. Se realizaron las descripciones de todas las variables por género, mediante los parámetros estadísticos correspondientes (medias aritméticas, desviación típica y error estándar para variables cuantitativas y frecuencias y 
porcentajes para las cualitativas). Las diferencias entre hombres y mujeres se analizaron mediante el test $t$ de Student y el test Chi-cuadrado $\left(\chi^{2}\right)$ para variables cuantitativas y cualitativas respectivamente. De acuerdo con el objetivo planteado se consideró como medida de salud objetiva el número de enfermedades diagnosticadas, y como medida de envejecimiento saludable un score obtenido con las variables salud percibida, satisfacción con la vida, estado de la boca y estado de la audición. Se aplicaron modelos de regresión lineal múltiple, con el fin establecer modelos predictivos multivariantes de envejecimiento saludable. El nivel de significación considerado es $p<0.05$.

\section{RESULTADOS}

Los resultados de las variables que se van a utilizar en los modelos de regresión aparecen en la tabla 1, donde se indican las diferencias significativas entre mujeres y hombres.

Tabla 1. Características de la muestra por género

\begin{tabular}{|c|c|c|c|}
\hline \multirow{2}{*}{ Variables } & Mujeres & Hombres & Test estadístico \\
\hline & Media $(S D)$ & Media $(S D)$ & \\
\hline Edad (años) & $73.9(5.8)$ & $74.8(6.6)$ & ns \\
\hline Años de educación & $6.6(4.9)$ & $7.5(4.4)$ & ns \\
\hline Edad ( años) de primera maternidad & $26.4(4.0)$ & -- & -- \\
\hline Número de descendientes & $2.5(1.5)$ & -- & -- \\
\hline Talla sentado $(\mathrm{cm})$ & $79.0(3.8)$ & $85.2(4.0)$ & $t=10.538: p=0.000$ \\
\hline Envergadura $(\mathrm{cm})$ & $154.4(6.4)$ & $168.3(10.0)$ & $t=10.605 ; p=0.000$ \\
\hline $\operatorname{IMC}\left(\mathrm{kg} / \mathrm{m}^{2}\right)$ & $29.3(4.2)$ & $29.1(3.6)$ & ns \\
\hline \multirow[t]{2}{*}{ Número de enfermedades } & $2.33(2.0)$ & $1.58(1.6)$ & $t=-2.703 ; p=0.007$ \\
\hline & $\%(\mathrm{~N})$ & $\%(\mathrm{~N})$ & \\
\hline \multicolumn{4}{|l|}{ Nivel de educación } \\
\hline Sin educación formal & $35.1(47)$ & $16.7(13)$ & \multirow{4}{*}{$\chi^{2}=8.593 ; p=0.035$} \\
\hline Primaria & $56.4(61)$ & $45.5(44)$ & \\
\hline Secundaria & $9.7(13)$ & $11.5(9)$ & \\
\hline Superior/universitaria & $9.7(13)$ & $15.4(12)$ & \\
\hline \multicolumn{4}{|l|}{ Cómo considera su salud } \\
\hline Mala & $12.8(17)$ & $7.7(6)$ & \multirow{4}{*}{ ns } \\
\hline Regular & $47.4(63)$ & $39.7(31)$ & \\
\hline Buena & $31.6(42)$ & $38.5(30)$ & \\
\hline Muy buena & $8.3(11)$ & $14.1(11)$ & \\
\hline \multicolumn{4}{|l|}{ Satisfacción vida } \\
\hline Muy poco & $2.5(3)$ & -- & \multirow{4}{*}{$\chi^{2}=10.421 ; p=0.015$} \\
\hline Algo & $24.6(29)$ & $8.3(6)$ & \\
\hline Bastante & $51.7(61)$ & $68.1(49)$ & \\
\hline Mucho & $21.2(25)$ & $23.6(17)$ & \\
\hline \multicolumn{4}{|l|}{ Como es su estado auditivo } \\
\hline Muy malo & $4.5(6)$ & $3.9(3)$ & \multirow{4}{*}{ ns } \\
\hline Malo & $23.3(31)$ & $26.0(20)$ & \\
\hline Bueno & $48.1(64)$ & $49.4(38)$ & \\
\hline Muy bueno & $23.3(31)$ & $20.8(16)$ & \\
\hline \multicolumn{4}{|l|}{ Como es el estado de su boca } \\
\hline Pobre & $49.6(66)$ & $39.0(30)$ & \multirow{4}{*}{ ns } \\
\hline Normal & $23.3(31)$ & $23.4(18)$ & \\
\hline Bueno & $19.5(26)$ & $29.9(23)$ & \\
\hline Muy Bueno & $7.5(10)$ & $7.8(6)$ & \\
\hline
\end{tabular}


Entre las variables estudiadas, observamos que las mujeres presentan un nivel de educación significativamente menor que los hombres. En cuanto a las variables antropométricas, las mujeres tienen, significativamente, un menor tamaño corporal que los hombres si se considera la talla y la envergadura. Por último, encontramos que son ellas las que menos satisfechas están con la vida y presentan un mayor número de enfermedades.

En la tabla 2 se presentan los resultados de los modelos de regresión lineal cuya variable dependiente es el número de enfermedades. Se presenta un primer modelo (Modelo 1) que incluye la variable género. Un segundo modelo (Modelo 2) realizado para hombres y mujeres por separado. Por último, en la misma tabla se presentan los resultados del modelo (modelo 3) ajustado por variables reproductivas generado en el caso de las mujeres donde se incluyen variables de patrones reproductores consideradas (edad de primera maternidad y número total de descendientes) (Tabla 2).

Tabla 2. Modelos predictivos para número de enfermedades

\begin{tabular}{|c|c|c|c|c|}
\hline \multicolumn{5}{|c|}{ Modelo 1. Ajustado por género, variables antropométricas y socioeconómicas } \\
\hline & Beta & Sig. & \multicolumn{2}{|c|}{ IC $95 \%$} \\
\hline Género (referencia=hombres) & 1.342 & $0.006^{*}$ & 0.392 & 2.292 \\
\hline Edad (años) & 0.031 & 0.283 & -0.025 & 0.087 \\
\hline Número de años de educación & -0.034 & 0.355 & -0.106 & 0.038 \\
\hline Talla sentado $(\mathrm{cm})$ & -0.077 & 0.117 & -0.174 & 0.020 \\
\hline Envergadura $(\mathrm{cm})$ & 0.045 & $0.041^{*}$ & 0.002 & 0.088 \\
\hline \multicolumn{5}{|c|}{ Modelo 2. Ajustado por variables antropométricas y socioeconómicas } \\
\hline Hombres & Beta & Sig. & \multicolumn{2}{|c|}{ IC $95 \%$} \\
\hline Edad (años) & 0.015 & 0.645 & -0.051 & 0.082 \\
\hline Número de años de educación & -0.037 & 0.459 & -0.137 & 0.063 \\
\hline Talla sentado $(\mathrm{cm})$ & -0.127 & 0.068 & -0.264 & 0.010 \\
\hline Envergadura $(\mathrm{cm})$ & 0.019 & 0.404 & -0.027 & 0.065 \\
\hline Mujeres & Beta & Sig. & \multicolumn{2}{|c|}{ IC $95 \%$} \\
\hline Edad (años) & 0.024 & 0.547 & -0.055 & 0.103 \\
\hline Número de años de educación & -0.037 & 0.432 & -0.130 & 0.056 \\
\hline Talla sentado $(\mathrm{cm})$ & -0.069 & 0.278 & -0.194 & 0.056 \\
\hline Envergadura $(\mathrm{cm})$ & 0.083 & $0.020^{*}$ & 0.013 & 0.152 \\
\hline \multicolumn{5}{|c|}{ Modelo 3. Ajustado por variables reproductivas, antropométricas y socioeconómicas } \\
\hline Mujeres & Beta & Sig. & \multicolumn{2}{|c|}{ IC $95 \%$} \\
\hline Edad (años) & 0.052 & 0.243 & -0.036 & 0.139 \\
\hline Número de años de educación & -0.004 & 0.939 & -0.116 & 0.107 \\
\hline Talla sentado $(\mathrm{cm})$ & -0.046 & 0.489 & -0.177 & 0.085 \\
\hline Envergadura $(\mathrm{cm})$ & 0.081 & $0.035^{*}$ & 0.006 & 0.156 \\
\hline Edad de primera maternidad & -0.124 & $0.044 *$ & -0.244 & -0.003 \\
\hline Número de hijos & -0.160 & 0.331 & -0.484 & 0.165 \\
\hline
\end{tabular}

Como se observa en la tabla 2, en el modelo 1 aparecen como variables predictivas el género y la envergadura, aunque con un coeficiente muy cercano a cero.

En cuanto al modelo 2, en los hombres, no se detecta que ninguna variable tenga carácter predictivo. En el caso de las mujeres aparece la envergadura. 
En el tercer modelo, realizado solo para las mujeres (Tabla 2), se incluyen la edad de primera maternidad y el número total de descendientes. La edad de primera maternidad influye negativa y significativamente sobre el número de enfermedades, es decir, una edad de primera maternidad más tardía se asocia a menor número de enfermedades en la última etapa del ciclo vital.

En la tabla 3 se muestran las variables predictivas de envejecimiento saludable. Se presentan los resultados de tres modelos como en la tabla anterior.

En el modelo 1 aparece como significativo el número de años de educación que se asocia positivamente con envejecimiento saludable, es decir, las personas con mayor nivel de estudios presentan mejor envejecimiento. Sin embargo al considerar solo a los hombres aparecen como variables predictivas la edad y la talla sentado (a menor edad y a menor talla sentado, mayor valor para envejecimiento saludable) y desaparece el efecto de los años de educación.

En el caso de las mujeres solo tiene efecto significativo el número de años de enseñanza reglada, es decir, las mujeres con más estudios tienen mejor envejecimiento. Sin embargo, cuando se introducen las variables edad de primera maternidad y número total de descendientes, desaparece la significación para el número de años de educación y solamente se mantiene la edad de primera maternidad (edades de maternidad más tardías se asocian a mayor puntuación en envejecimiento activo).

Tabla 3. Modelos predictivos para envejecimiento saludable

\begin{tabular}{|c|c|c|c|c|}
\hline \multicolumn{5}{|c|}{ Modelo 1. Modelo ajustado por género, variables antropométricas y socioeconómicas } \\
\hline & Beta & Sig. & \multicolumn{2}{|c|}{ IC $95 \%$} \\
\hline Género (referencia=hombres) & -0.554 & 0.372 & -1.777 & 0.670 \\
\hline Edad (años) & -0.061 & 0.093 & -0.132 & 0.010 \\
\hline Número de años de educación & $0.127 *$ & 0.006 & 0.036 & 0.218 \\
\hline Talla sentado $(\mathrm{cm})$ & -0.005 & 0.935 & -0.124 & 0.114 \\
\hline Envergadura $(\mathrm{cm})$ & 0.002 & 0.943 & -0.053 & 0.057 \\
\hline \multicolumn{5}{|c|}{ Modelo 2. Modelos ajustados por variables antropométricas y socioeconómicas } \\
\hline Hombres & Beta & Sig. & \multicolumn{2}{|c|}{ IC $95 \%$} \\
\hline Edad (años) & -0.127 & $0.022 *$ & -0.234 & -0.019 \\
\hline Número de años de educación & 0.120 & 0.144 & -0.043 & 0.284 \\
\hline Talla sentado $(\mathrm{cm})$ & -0.214 & $0.024 *$ & -0.399 & -0.030 \\
\hline Envergadura $(\mathrm{cm})$ & 0.028 & 0.438 & -0.045 & 0.101 \\
\hline Mujeres & Beta & Sig. & \multicolumn{2}{|c|}{ IC $95 \%$} \\
\hline Edad (años) & -0.016 & 0.733 & -0.112 & 0.079 \\
\hline Número de años de educación & 0.124 & $0.027 *$ & 0.015 & 0.233 \\
\hline Talla sentado $(\mathrm{cm})$ & 0.101 & 0.194 & -0.052 & 0.255 \\
\hline Envergadura $(\mathrm{cm})$ & -0.028 & 0.527 & -0.114 & 0.059 \\
\hline \multicolumn{5}{|c|}{ Modelo 3. Modelo ajustado por variables reproductivas, antropométricas y socioeconómicas } \\
\hline & Beta & Sig. & \multicolumn{2}{|c|}{ IC $95 \%$} \\
\hline Edad (años) & -0.015 & 0.756 & -0.114 & 0.083 \\
\hline Número de años de educación & 0.078 & 0.213 & -0.045 & 0.201 \\
\hline Edad de primera maternidad & 0.147 & $0.039 *$ & 0.008 & 0.286 \\
\hline Número de hijos & -0.212 & 0.244 & -0.573 & 0.148 \\
\hline Talla sentado $(\mathrm{cm})$ & 0.125 & 0.105 & -0.027 & 0.277 \\
\hline Envergadura $(\mathrm{cm})$ & -0.014 & 0.737 & -0.100 & 0.071 \\
\hline
\end{tabular}




\section{DISCUSIÓN}

Los resultados muestran diferentes variables predictivas de envejecimiento saludable en las personas mayores.

El sexo de los individuos es una variable muy relacionada con la salud diagnosticada, con peores resultados entre las mujeres, como ha sido ampliamente referido en la literatura (Crimmins et al., 2011; De Angelis et al., 2011; Montero et al., 2013).

En este estudio, las mujeres tienen mayor número de enfermedades diagnosticadas. Sin embargo, y a diferencia de las investigaciones que incluyen la perspectiva de género (Crimmins et al., 2011; Acevedo y López, 2013) el envejecimiento saludable no se ve afectado por este factor.

$\mathrm{Al}$ igual que en otros estudios otra variable de clara influencia sobre el tipo de envejecimiento es el nivel de estudios (Moe y Hagen, 2011). El indicador analizado como envejecimiento saludable aparece asociado de manera positiva al número de años de escolarización, cuándo se considera el total de la muestra. En nuestro estudio es de destacar que cuándo se realiza un análisis de esta asociación controlando la variable género, desaparece el efecto de la educación en los hombres, pero se mantiene en las mujeres.

Investigaciones recientes (Montero et al., 2011) muestran la influencia de las primera fases del ciclo vital y de los factores socio-económicos de esas etapas sobre el envejecimiento saludable (Lavery y Lee, 2009; Adams, 2004). La envergadura o la talla sentado aportan información importante sobre cómo han influido los factores nutricionales y socioeconómicos en el proceso de crecimiento y desarrollo de los individuos (Montero et al., 2013). Nuestros resultados apoyan también esta idea, mejores condiciones de vida durante la niñez y la adolescencia (mayor nivel de estudios), se asocian con mejor envejecimiento.

En contra de lo esperado, la envergadura, utilizada en lugar de la talla para soslayar el problema de la disminución en estatura asociado al envejecimiento, se asocia, en la muestra estudiada con un mayor número de enfermedades, es decir, a mayor estatura (mejores condiciones durante el proceso de crecimiento y desarrollo), peor envejecimiento. Esto puede ser debido a limitaciones metodológicas del estudio ya que se trata de un estudio transversal que impide valorar el proceso de reducción de la estatura asociado a involución senil. Convendría investigar sobre la conveniencia de utilizar otros indicadores de estatura en las investigaciones con personas mayores, como la altura de la rodilla, etc. No obstante, en los hombres si se observa que una menor talla sentado (buenas condiciones de crecimiento) es predictiva de un envejecimiento más saludable. 
Este estudio también apoya el hecho de que en los hombres y en las mujeres actúan factores diferentes a lo largo de su vida que van a influir de manera diferencial en su forma de envejecer (Lavery y Lee, 2009). En las mujeres, edades tempranas de maternidad se asocian a mayor número de enfermedades y a envejecimiento menos saludable.

En conclusión, los resultados de este estudio inciden en la importancia de la perspectiva de género y del impacto de factores del pasado sobre la forma de envejecer de los adultos mayores.

\section{Agradecimientos}

Al Ministerio de Sanidad, Política Social e Igualdad. El género como unidad de análisis en el estudio de los condicionantes bioculturales del envejecimiento saludable (Q2818013A), 2011-2014.

\section{REFERENCIAS}

Acevedo, P. y López, M.R. (2013). Percepción del estado de salud en mujeres y hombres de edad avanzada En P. Folguera, V. Maquieira, M.J. Matilla, P. Montero y M.J Vara (Eds.), Género y Envejecimiento (pp. 362-376). Madrid: Ediciones de la Universidad Autónoma de Madrid.

Adams, J.M. y Whithe, M. (2004). Biological ageing. A fundamental, biological link between socio-economic status and health? European Journal of Public Health, 14, 331-334.

Crimmins, E.M., Kim, J.K. y Solé-Auró, A. (2011). Gender differences in health: results from SHARE, ELSA and HRS. European Journal Public Health, 21(1), 81-91.

Christensen, K., Doblhammer, G., Rau, R. y Vaupel, J.W. (2009). Ageing populations: the challenges ahead. Lancet, 374, 9696, 1196-1208.

De Angelis, C.D. y Winker, M.A. (2001). Women's health-filling the gaps. JAMA, 285, 15081509.

Gularnik, J., Butterworth, S., Wadsworth, M. y Kuh, M. (2006). Childhood socioeconomic status predicts physical functioning a half century later. Journal of Gerontology Medical Science, 61(7), 694-701.

Hayward, M. y Gorman, B. (2004). The long arm of childhood: the influence of early-life social conditions on men mortality. Demography, 41, 87-107.

Hank, K. (2011). "How 'successful' do older Europeans age? Findings from SHARE”. Journal of Gerontology: Social Sciences, 66B, 230-236.

Lavery, D. y Lee, M. (2009). Effects of early life on elderly health. Today's Research on Ageing, $16,1-5$.

Moe, J. y Hagen, T. (2011). Trends and variation in mild disability and functional limitations among older adults in Norway, 1986-2008. European Journal Ageing, 8, 49-61.

Montero, M.P. y Rodríguez, S. (2013). Indicadores de envejecimiento activo en la población española. En Folguera P., Maquieira V., Matilla M.J., Montero P., Vara M.J. (Eds.), Género y Envejecimiento (pp. 313-333). Madrid: Ediciones de la Universidad Autónoma de Madrid. 
Montero-López, M.P., Fernández-Ballesteros, R., Mora-Urda, A.I. y Romero-Collazos, J.F. (2013). Nutrition and active ageing in a Spanish sample. Revista Española de Antropología Física, 34, 27-34.

Montero, M.P., Fernández-Ballesteros, R., Zamarrón, M.D. y Rodríguez, S. (2011). Anthropometric, Body Composition and Health Determinants of Active Ageing: A Gender Approach. Journal of Biosocial Sciences, 43, 597-610.

Salomon, J.A., Wang, H., Freeman, M.K., Vos, T., Flaxman, A.D., Lopez, A.D. y Murray, C.J.L. (2013). Healthy life expectancy for 187 countries, 1990-2010: a systematic analysis for the Global Burden Disease Study 2010. Lancet, 380, 9859, 2144-2162.

Vaupel, J.W. (2010). Biodemography of human ageing. Nature, 464, 25, 536-542.

WHO (2002) Active ageing. A Policy Framework. WHO, Geneve.

Recibido: 26 de abril de 2014 Recepción Modificaciones: 26 de mayo de 2014 Aceptado: 15 de junio de 2014 